\title{
Panorama Measurement Based on Spherical Projective Geometry
}

\author{
Shuai Liu, Lingli Zhao ${ }^{1}$ and Junsheng Li \\ School of Engineering, Honghe University, Mengzi, China, 661100, \\ zll_csu@126.com \\ Li MA \\ Changjiang Institute of Survey, Planning, Design and Research, Wuhan, Hubei, \\ China, 430010
}

\begin{abstract}
Previous panoramic studies focused on image stitching and the panoramic camera development. In recent years, people began to focus on panoramic scalability issues, the existed methods adopt traditional photogrammetric methods for data processing based on a multi-directional panorama images, using a large number of control points and combined bundle adjustment. Indeed, panorama itself is a kind modeling method, it is completely unnecessary to reconstruct the panoramic entire scene for measurement. So in this paper, we focus on a kind of panorama measurement from spherical projective geometry we studied and constructed the relative, absolute orientation and measurable algorithms based on spherical stereo pair panorama in order to achieve panoramic measurement in the process of panorama roaming. The experiment shows that the approach is much validated, and something useful is obtained.
\end{abstract}

Keywords: Panorama, 3D Reconstruction, Spherical Projective Geometry, image

\section{Introduction}

Three-dimensional modeling is one of the core issues in three-dimensional Geographic Information System (3D GIS). In summary, 3D modeling methods can be generally summed up in two categories: the ones is that digital elevation models combined with images to build on 2.5-dimensional digital terrain model; the other is that the use of the block model combined with texture to build multiple levels of detail models (Levels of detail, LOD) of the three-dimensional model (such as CityGML). CityGML is an international standard for the representation and exchange of semantic 3D city and landscape models adopted by the Open Geospatial Consortium (OGC) [1,2,3,4], which can construct and display not only the urban model and surface appearance, also involve semantics, thematic attribute expression, classification and collection. CityGML is utilized for use in many applications, including but not limited to urban planning, facility management, disaster management, homeland security, personal navigation, etc. For example, how an emergency operator can find a suitable location and building for a field hospital after the explosion of a dirty bomb [5]. CityGML can provide important information for disaster management [6], which includes emergency route planning and indoor navigation [7-8].

However, there are some limitations by using these two methods to construct threedimensional models; the former is low currency and lacking of enough texture details, the later is the complex construction technologies and difficulty updating, often cannot satisfy the demand for emergency disposal or real-time application process on the local highdetail, and the currency three-dimensional scene.

\footnotetext{
${ }^{1}$ Corresponding author: Lingli ZHAO, $\mathrm{PhD}$, interest covers 3D modeling, data integration and data mining. zll_csu@126.com
} 
Google Earth is a typical application of the related fields. The use of modeling techniques in addition to the general three-dimensional modeling mentioned above, it introduces a kind of panoramic model, namely Street View. The introduction of the Street View is to solve the traditional three-dimensional expression of inadequate authenticity, difficult data acquisition as well as laborious and time-consuming modeling.

Previous panoramic studies focused on image stitching [9-10] and the panoramic camera development [11-12]. In recent years, people began to focus on panoramic scalability issues, Luhmann discussed panorama reconstruction based on cylindrical projection [13-15], Fangi studied the panoramic modeling from spherical projection [1618].These methods adopt traditional photogrammetric methods for data processing based on a multi-directional panorama images, using a large number of control points, combined bundle adjustment in order to achieve three-dimensional reconstruction.

Indeed, panorama itself is a kind modeling method. Its main function is the browser landscape. However, it is completely unnecessary to reconstruct the panoramic entire scene for measurement. Our key consideration is how to provide scalability for panorama by human real-time interaction to measure the object of interest in the panoramic roaming.

Therefore, we studied and constructed the relative, absolute orientation and measurable algorithms based on spherical stereo pair panorama in order to achieve panoramic measurement in the process of panorama roaming.

Therefore, we studied and constructed the relative, absolute orientation and measurable algorithms based on spherical stereo pair panorama in order to achieve panoramic measurement in the process of panorama roaming.

\section{Spherical Geometric Modeling of Panorama}

Panoramic modeling is designed to provide users with a full 360-degree landscape viewing and observation. Spherical panoramic is a kind of sphere model. The observation point is located in the center of the sphere, seen in Figure 1.

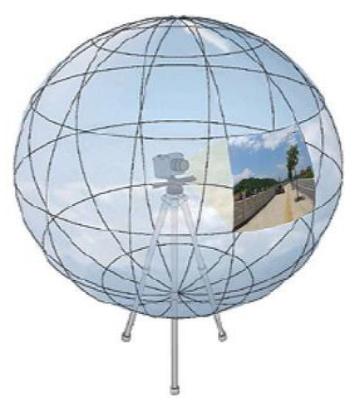

\section{Figure 1. Diagram of Spherical Panorama Modeling}

The construction method of sphere panoramic model is shown in Figure 2. From a unique station point, images -partly overlapping - are taken around to cover totally or partially the horizon. The images are then stitched together and projected on a virtual sphere whose radius $\mathrm{R}$ is arbitrary but equal to the focal length of the camera in case we don't want to reduce the original resolution. The projection centre and the focal length have to remain constant during the taking phase. The sphere is then mapped in a cartographic plane with the so-called equirectangular projection or azimuthzenith projection. 

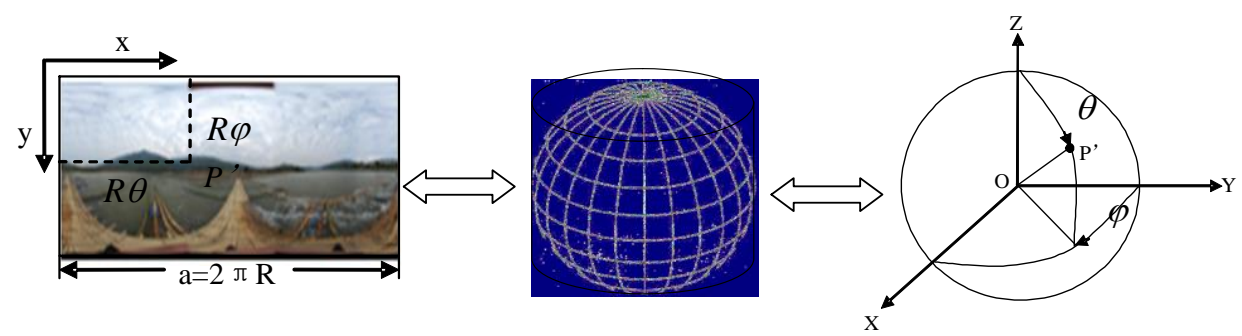

Figure 2. Mapping Relation Between Panorama Image and Spherical Geometry Model

Set panoramic image length is a, the spherical model of radius is $\mathrm{R}$, after the image is mapped, the relationship between the $\mathrm{R}$ and a should bea $=2 \pi \mathrm{R}$, so the aspect ratio of the panoramic image should be $2: 1$. Set pixel coordinates of panoramic images are $p^{\prime}(x, y)$, corresponding 3D point coordinates on the sphere model are $\mathrm{P}^{\prime}(\mathrm{X}, \mathrm{Y}, \mathrm{Z})$, the mapping relationship according to the literature [11] shown in Figure 2 is as follows:

$$
\begin{aligned}
& \left\{\begin{array}{l}
x=R \cdot \theta \\
y=R \cdot \varphi \\
R=a / 2 \pi
\end{array}\right. \\
& \left\{\begin{array}{l}
X=R \cdot \sin \theta \cdot \sin \varphi \\
Y=R \cdot \cos \theta \cdot \sin \varphi \\
Z=R \cdot \cos \varphi
\end{array}\right.
\end{aligned}
$$

Where the angles of $\theta$ and $\varphi$ are radians values.

\section{The Construction of Three-Dimensional Panoramic Measurement Algorithms}

This section constructed the following four algorithms to complete spherical panoramic three-dimensional modeling to achieve the scene measurements. The algorithms and the relationship are shown in Figure 3.

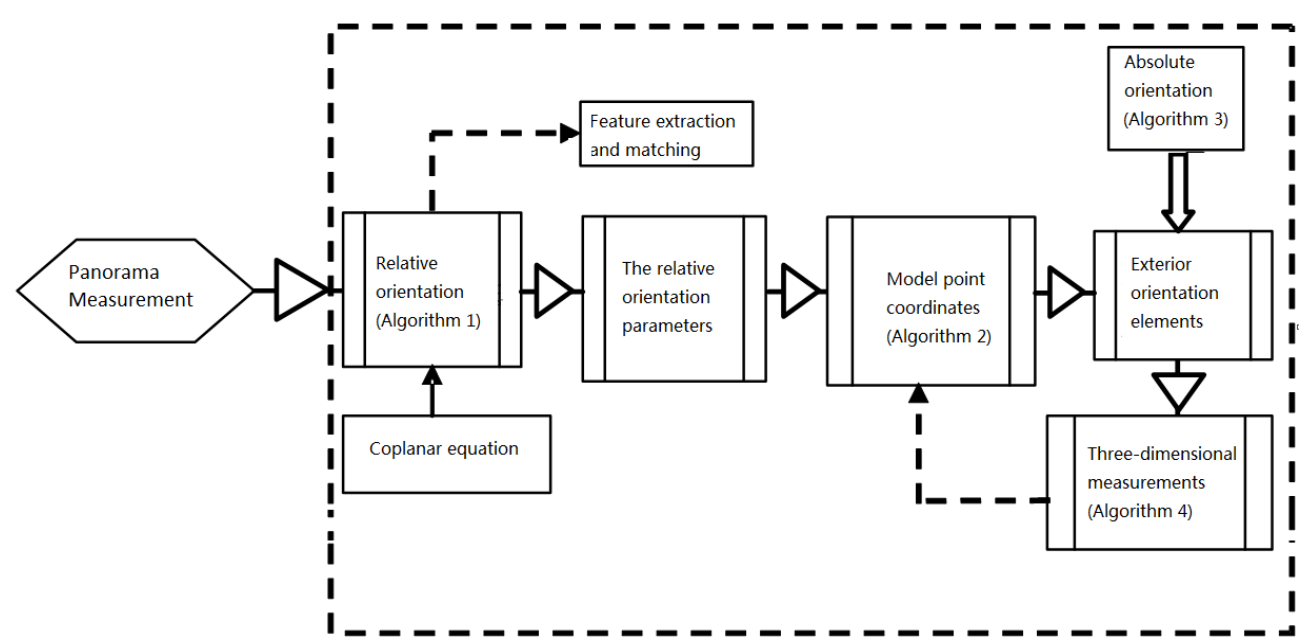

Figure 3. The Diagram of 3D Reconstruction Approach Containing Several Algorithms Based on two Panoramic Images 


\subsection{Relative Orientation and Absolute Orientation Algorithms}

Algorithm 1

Relative orientation: Calculate the parameters of relative orientation and attitude between two panoramic images.

Conditions: Given more than five corresponding points' pixel coordinates between two panoramas.

Target: the parameters of relative orientation and attitude between two panoramic images. The flow chart shown is in Figure 4, the specific steps as follows:

a) Determine the initial values of relative orientation, $\mu=v=\varphi=\omega=\kappa=0$, where $\mu$ and $\nu$ are the corresponding angles of the baselines $\mathrm{By}$ and $\mathrm{Bz}$ respectively,. $\varphi, \omega$ and $\kappa$ are relative to the rotation angles respectively.

b) According to the equation(3) the image plane pixel $(\mathrm{x}, \mathrm{y})$ is converted to spherical coordinates (X, Y, Z);

c) Exterior orientation parameters of the camera lens have small changes in the data collection, so the error equation is constructed according to the formula (4);

$$
F=F^{0}+\frac{\partial F}{\partial \mu} \Delta \mu+\frac{\partial F}{\partial v} \Delta v+\frac{\partial F}{\partial \varphi} \Delta \varphi+\frac{\partial F}{\partial \omega} \Delta \omega+\frac{\partial F}{\partial \kappa} \Delta \kappa=0
$$

d) Relative orientation elements accumulated by corrections, calculating the coefficients and the constanst of the error equation;

e) Solve the normal equation and the relative orientation element corrections;

f) Determine whether the absolute value corrections are less than the threshold, if greater than the threshold, then skip to step d), otherwise, step g);

g) Output the relative orientation elements $(\mu, \nu, \varphi, \omega, \kappa)$.

Algorithm 2

Model coordinates: Calculate the model coordinates according to the known relative orientation elements in the local sphere coordinates system.

Conditions: Given relative orientation elements and $\mathrm{N}$ corresponding points' pixel coordinates between two panoramas.

Target: the local sphere coordinates of each corresponding point (model coordinates). The flow chart shown is in Figure 5, the specific steps as follows:

a) Each point requires the step b) and step c);

b) The intermediate coefficients of $\mathrm{N} 1$ and $\mathrm{N} 2$ are required according to the rotation matrix $\mathrm{R}$ based on the relative orientation;

c) Calculate model point coordinates;

d) Output model points coordinates

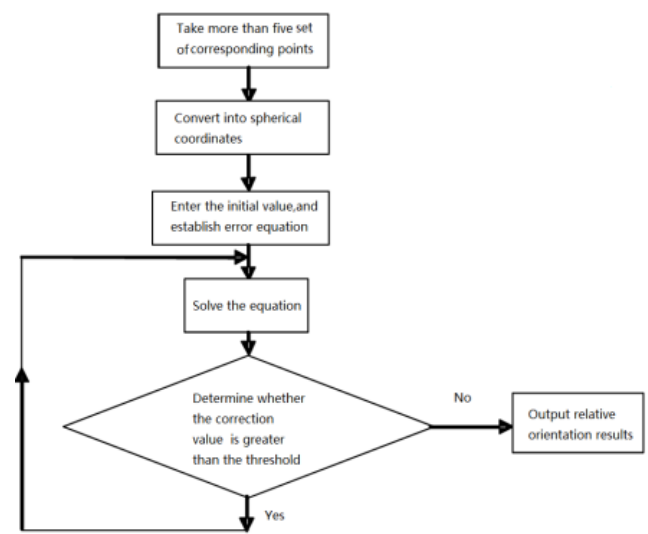

Figure 4. The Flow Chart of the Relative Orientation 


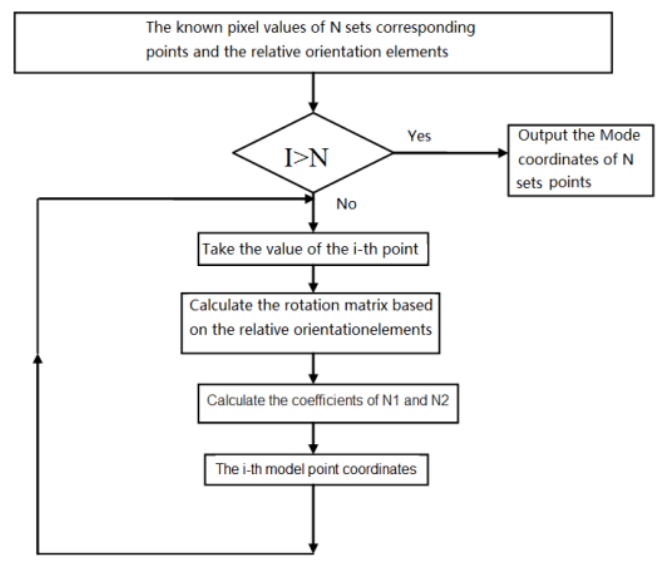

Figure 5. The Flow Chart of Model Point Coordinates Calculation

Algorithm 3

Absolute orientation: Calculate the conversion relationship from the mode points' coordinates to the object coordinates.

Conditions: Given more than three points of the mode points' coordinates and the object coordinates.

Target: Calculate the exterior orientation elements of absolute orientation. The flow chart shown is in Figure 6, the specific steps as follows:

a) determine the initial exterior orientation elements of the absolute orientation, $\phi=0$, $\Omega=0, K=0, \lambda_{0}=1, X_{0}=0, Y_{0}=0, Z_{0}=0$, where $(\phi, \Omega, K)$ are the parameter of rotation $s$ angles, $\left(X_{0}, Y_{0}, Z_{0}\right)$ are translation parameters;

b) The two sets coordinates of points is centralized, and calculate the centralized coordinates;

c) The error equation is constructed according to the formula (5);

$F=F^{0}+\frac{\partial F}{\partial \lambda_{0}} \Delta \lambda_{0}+\frac{\partial F}{\partial \Phi} \Delta \Phi+\frac{\partial F}{\partial \Omega} \Delta \Omega+\frac{\partial F}{\partial K} \Delta K+\frac{\partial F}{\partial X_{0}} \Delta X_{0}+\frac{\partial F}{\partial Y_{0}} \Delta Y_{0}+\frac{\partial F}{\partial Z_{0}} \Delta Z_{0}$

d) Exterior orientation elements accumulated by corrections, calculating the coefficients and the constants of the error equation;

e) Solve the normal equation and the absolute orientation element corrections;

f) Determine whether the absolute value corrections are less than the threshold, if greater than the threshold, then skip to step d), otherwise, step g);

g) Output the absolute orientation elements $(\phi, \Omega, \mathrm{K}, \lambda 0, \mathrm{X} 0, \mathrm{Y} 0, \mathrm{Z} 0)$.

\subsection{The Measurement Algorithm}

\section{Algorithm 4}

Algorithm 4 is the measurement algorithm. The main application is to achieve realtime computing by orientation relationship between panoramic images.

Panoramic measurement: deduce object coordinates from the selected pixel coordinates of points in the two panoramic images.

Conditions: Given the relative orientation elements and the absolute orientation exterior elements.

Target: Select point calculated in the panoramic image and the corresponding point. The pixel coordinates of the selected point in the two images is converted to spherical coordinates, and calculate the model point coordinates by the relative orientation elements, then calculate objects coordinates according to the absolute orientation coefficient. The flow chart shown is in Figure 7, the specific steps as follows: 
a) Select the point you want to calculate in the reference panoramic image, and select the corresponding point in another panorama image.

b) The image pixel coordinates of the two selected points in the panorama are converted to spherical coordinates;

c) Calculate the model point coordinates by calling algorithm 2;

d) Calculate the rotation and translation parameters according to the absolute orientation elements;

e) Calculate the object space coordinates;

f) Determine whether the need to calculate the other points, If yes, skip to step a), otherwise, step g);

g) According to the calculated object coordinates, a simple measurement can be performed, such as calculating the distance.

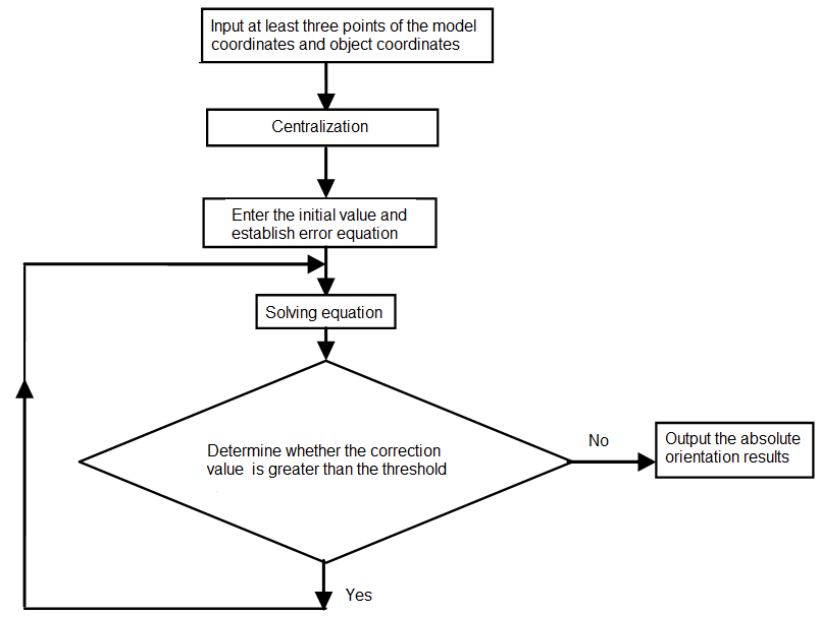

Figure 6. The Flow Chart of the Absolute Orientation

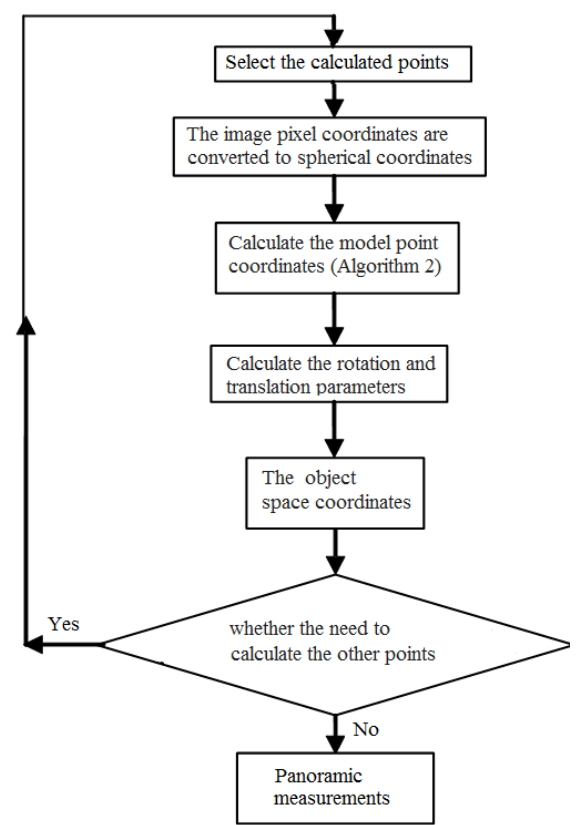

Figure 7. The Flow Chart of Panoramic Measurement

It is noteworthy that there is a little slight rotation in the absolute orientation between the two panoramas. It can consider the establishment of coplanar equations 
by corresponding points. Moreover, the control points can be obtained by the known information in the scene, thus constructing object coordinates.

\section{Experiments and Analysis}

In order to verify the method proposed by the paper, we use high resolution digital camera to obtain different positions of the two sets of 360 degree image sequence in a landmark, the two panoramic images are obtained by stitching as shown in Figure 8 Red dot represents the two stations, and the length of baseline is about $7 \mathrm{~m}$.

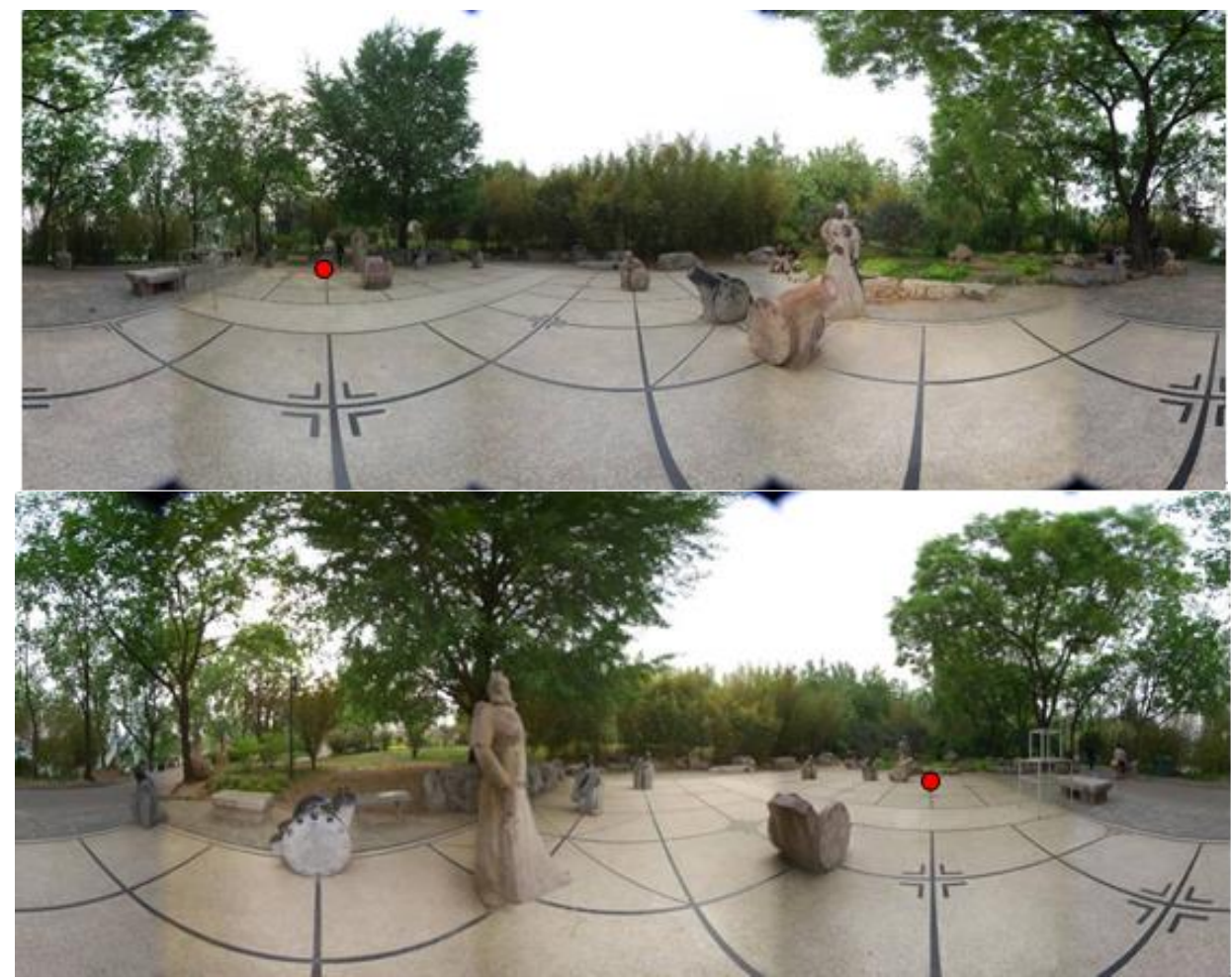

Figure 8. Panoramic Stereo Pair Acquisition

\subsection{The Time Complexity and Space Complexity Analyses of the Algorithms}

The main title (on the first page) should begin $13 / 16$ inches ( 7 picas) from the top edge of the page, centered, and in Times New Roman 14-point, boldface type. Capitalize the first letter of nouns, pronouns, verbs, adjectives, and adverbs; do not capitalize articles, coordinate conjunctions, or prepositions (unless the title begins with such a word). Please initially capitalize only the first word in other titles, including section titles and first, second, and third-order headings (for example, "Titles and headings" — as in these guidelines). Leave two blank lines after the title.

There are each time complexity and space complexity given below. The running time is shown in Table 1. Test equipment is the ordinary computer, CPU is dual-core, $2.8 \mathrm{GHz}$, RAM is 2GB; experimental environment is Matlab 7.1.

Algorithm 1: Set the number of iterations in the calculation is $M$, iterative calculation step is $\mathrm{n} 1$. The total run time $=\mathrm{n} 1 * \mathrm{M}$; auxiliary space occupied $=\mathrm{O}(5)$.

Algorithm 2: Set the number of the model points is $\mathrm{N}$. Total run time $=\mathrm{N}$; auxiliary space occupied $=\mathrm{O}(1)$. 
Algorithm 3: Set the number of iterations in the absolute orientation is $\mathrm{K}$, iterative calculation step is $\mathrm{n} 2$. The total run time $=\mathrm{n} 2 * \mathrm{~K}$; auxiliary space occupied $=\mathrm{O}(6)$.

Algorithm 4: Set the number of points to calculate the object coordinates is $\mathrm{P}$, the calculation step is $n 3$. The total run time $=P^{*}(n 3+K)$, auxiliary space occupied $=O(3)$.

Table 1. Experimental Run Schedule

\begin{tabular}{ccccc}
\hline Algorithm name & Algorithm 1 Run time & $\begin{array}{c}\text { Algorithm 2 } \\
\text { Run time }\end{array}$ & $\begin{array}{c}\text { Algorithm 3 } \\
\text { Run time }\end{array}$ & $\begin{array}{c}\text { Algorithm 4 } \\
\text { Run time }\end{array}$ \\
\hline Time (Second) & 0.1 & 0.03 & 0.2 & 0.6 \\
\hline
\end{tabular}

\subsection{Algorithm Implement}

Establish the object coordinate system by arranging the orientation control device shown on the right of Figure 9, and select 15 lines in the scene to be examined in Figure 9, and the results are showed in Table 2. After relative orientation - absolute orientation and through the open source PTViewer [19] panorama roaming is implemented in the Java environment, and the orientation parameters attached to Web environment to achieve panoramic measurement. The process is shown in Figure 10, the upper part shows the top of a sculpture measurement process, the yellow circle is the point the user has chosen on the one panorama, the yellow circle below is the corresponding point..

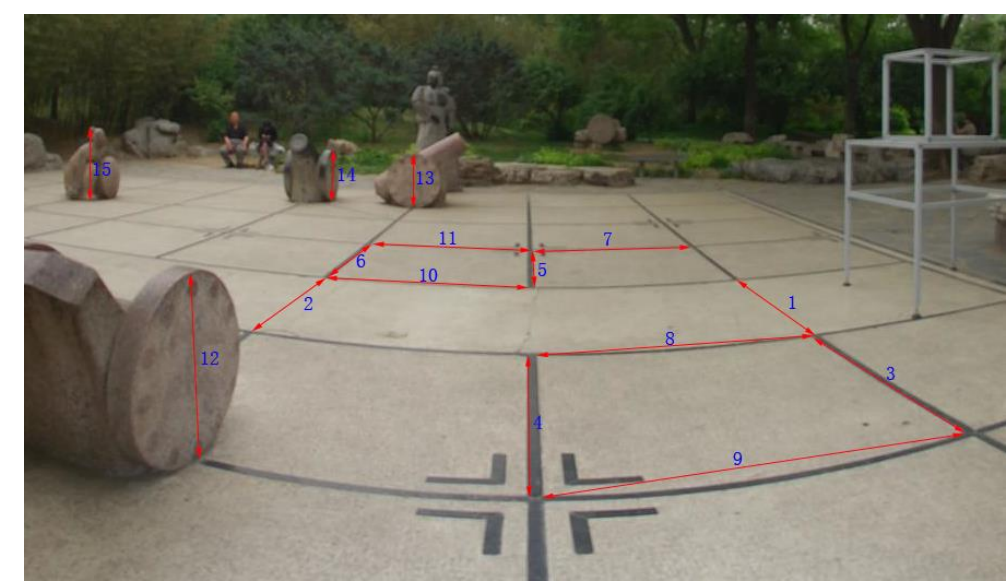

Figure 9. Control Points, Check Lines and the Coordinates Construction

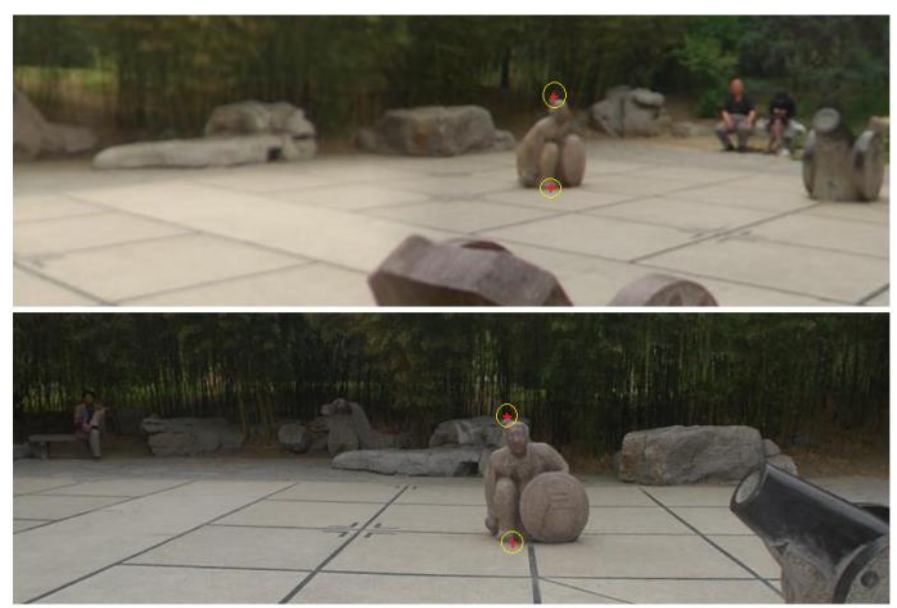

Figure 10. The Process of Panoramic Measurement 
Table 2. Accuracy of Each Distance for Check Lines

\begin{tabular}{|c|c|c|c|c|c|c|c|c|}
\hline Check lines & 1 & 2 & 3 & 4 & 5 & 6 & 7 & 8 \\
\hline $\begin{array}{l}\text { Actual } \\
\text { length /cm }\end{array}$ & 145.0 & 145.3 & 140.0 & 140.1 & 140.2 & 139.9 & 140.0 & 140.4 \\
\hline $\begin{array}{l}\text { Calculation } \\
\text { length /cm }\end{array}$ & 138.3 & 138.2 & 135 & 136 & 136 & 135.5 & 141.2 & 139.9 \\
\hline $\begin{array}{l}\text { Absolute } \\
\text { error /cm }\end{array}$ & 6.7 & 7.1 & 5.0 & 4.1 & 4.2 & 4.4 & -1.2 & 0.5 \\
\hline $\begin{array}{l}\text { Relative } \\
\text { error }\end{array}$ & 0.046 & 0.049 & 0.021 & 0.029 & 0.030 & 0.031 & 0.009 & 0.004 \\
\hline check lines & 9 & 10 & 11 & 12 & 13 & 14 & 15 & \\
\hline $\begin{array}{l}\text { Actual } \\
\text { length /cm }\end{array}$ & 140.1 & 140.0 & 140.1 & 73.1 & $0 \quad 72$. & 73.0 & 103.3 & \\
\hline $\begin{array}{l}\text { Calculate } \\
\text { length } / \mathrm{cm}\end{array}$ & 136.6 & 141 & 138.6 & 69 & 69. & $\begin{array}{ll} & 69 . \\
1\end{array}$ & 96.4 & \\
\hline $\begin{array}{l}\text { Absolute } \\
\text { error /cm }\end{array}$ & 3.5 & -1.0 & 1.5 & 4.1 & 2.3 & 3.9 & 6.9 & \\
\hline $\begin{array}{l}\text { Relative } \\
\text { error }\end{array}$ & 0.025 & 0.007 & 0.011 & 0.056 & 0.032 & 0.053 & 0.067 & \\
\hline
\end{tabular}

Matching points in relative orientation process are distributed throughout the scene, while matching points in the absolute orientation process are distributed around the orientation control device. The control points should theoretically be uniformly distributed throughout the scene, however, it will time-consuming, labor-intensive and impractical. Generally speaking, the closer the distance to the orientation control device where measurement accuracy is higher, the lower on the contrary. The same direction the accuracy of line segment 1 is better than the line segment 2 as shown in Table 2 .

\section{Conclusion}

The experiment reflects that the measurement method based on stereo panoramas in this paper has achieved certain accuracy. Though there is still a large gap on accuracy and precision in the field of survey, the method can quickly and easily provide users with certain metric information, for many applications(such as emergency rescue, police, outdoor visits, etc.) to provide great convenience.

In order to get an accurate measurement results, the next work includes the following two aspects:

(1) Error equation. The measurement method proposed by the paper is affected by many factors, such as feature extraction, image resolution, and control point distribution, how the error propagates.

(2) Automation. Increase automation modeling, and accelerate reconstruction time.

\section{Acknowledgement}

This research is supported by the National Natural Science Foundation (No. 41201418, 41301442), and Pecuniary aid of Yunnan Province basic research for application (2013fz127).

\section{References}

[1] J. Teller, A. K. Keita, C. Roussey and R. Laurini, "Urban Ontologies for an improved communication in urban civil engineering projects", In: Proceeding of the International Conference on Spatial Analysis and GEOmatics, Research \& Devel-opments, SAGEO 2005 Avignon, France, June, 20th-23 ${ }^{\text {rd }}$, (2005).

[2] A. Hamilton, H. Wang, A. M. Tanyer, Y. Arayici, X. Zhang and Y. Song, "Urban information model for city planning", ITcon, Special Is-sue From 3D to nD modelling, vol. 10, (2005). 
[3] G. Gröger, T. H. Kolbe, A. Czerwinski and C. Nagel, "OpenGIS City Ge-ography Markup Language (CityGML) Encoding Standard”, Version 1.0.0, OGC Doc. No. 08-007r1, Open Geospatial Consortium, (2008).

[4] "CityGML Homepage: http://www.citygml.org Featherstone WE, Sproule DM", Fitting AUSGeoid98 to the Australian Height Datum using GPS data and least squares collocation: application of a crossvalidation technique,Survey Review, vol. 38, no. 301, (2006), pp. 573-582.

[5] A. Lapierre and P. Cote, "Using Open Web Services for urban data man-agement: A testbed resulting from an OGC initiative for offering standard CAD/GIS/BIM services", In: Coors, V., Rumor, M., Fendel, E. M., Zlatanova S. (eds): Urban and Regional Data Management. Proceedings of the 26th UDMS, October 10-12, 2007, Stuttgart, Taylor \& Francis, (2007).

[6] T. H. Kolbe, G. Gröger and L. Plümer, „CityGML - 3D City Models and their Potential for Emergency Response", In: Zlatanova, Li (eds.), Geospatial Information Technology for Emergency Response, Taylor \& Francis, (2008).

[7] J. Lee and S. Zlatanova, "A 3D data model and topological analyses for emergency response in urban areas”, In: Zlatanova, Li (eds.), Geospatial In-formation Technology for Emergency Response, Taylor \& Francis, (2008).

[8] S. Mäs, W. Reinhardt and F. Wang, „Conception of a 3D Geodata Web Service for the Support of Indoor Navigation with GNSS", In: Proceeding of 3D GeoInfo 2006 in Kuala Lumpur, LNG\&C, Springer, (2006).

[9] R. Szeliski and H. Shum, "Creating full view panoramic image mosaics and environment maps", Proceeding of SIGGRAPH, (1997), pp. 251-258.

[10] B. Matthew and G. L. David, "Automatic panoramic image stitching using invariant features", International Journal of Computer Vision, no. 74, (2007), pp. 59-73.

[11] J. A. Parian, "Sensor modeling, camera calibration and point positioning with terrestrial panoramic cameras", Zurich: ETH, (2007).

[12] J. A. Parian and A. Gruena, "Sensor modeling, self-calibration and accuracy testing of panoramic cameras and laser scanners", ISPRS Journal of Photogrammetry and Remote Sensing, vol. 65, no. 1, (2010), pp. 60-76.

[13] T. Luhmann and W. Tecklenburg, "Bundle orientation and 3-D object reconstruction from multiplestation panoramic imagery", ISPRS Symposium, no. 5, (2002), pp. 181-186.

[14] T. Luhmann and W. Tecklenburg, "3-D object reconstruction from multiple-station panorama imagery", International Archives of the Photogrammetry, Remote Sensing and Spatial Information Sciences, no. 34, (2004), pp. 5-10.

[15] T. Luhmann, "Panorama photogrammetry for architectural applications", Mapping, no. 1, (2010), pp. 40-45.

[16] G. Fangi, "The multi-image spherical panoramas as a tool for architectural survey", XXI International CIPA Symposium, Athens, Greece, (2007), pp. 311-316.

[17] G. Fangi, "Further developments of the spherical photogrammetry for cultural heritage", 22nd CIPA Symposium, Kyoto, Japan, (2009), pp. 1-6.

[18] G. Fangi, "Multiscale multiresolution spherical photogrammetry with long focal lensed for architectural surveys", International Archives of Photogrammetry, Remote Sensing and Spatial Information Sciences, Part 5 Commission V Symposium, Newcastle upon Tyne, UK, vol. 37, (2010), pp. 1-6.

[19] http://webuser.fh-furtwangen.de/\%7Edersch/PTVJ/doc.html

\section{Authors}

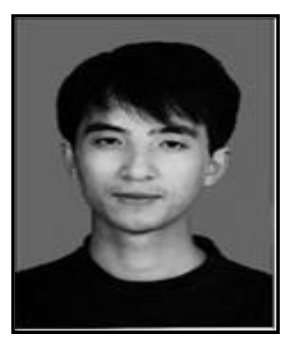

Shuai Liu, He received the B.S., M.S. and PhD degrees in Geographic Information System from Central South University, Changsha, China, in 2003, 2006 and 2011 respectively. From Match 2007 to May 2011, he was a research associate at National Geomatics Center of China. Currently, he is working at School of Engineering of Honghe University, Mengzi, China. His research interest covers computer vision, 3D modeling and virtual augmented reality. 

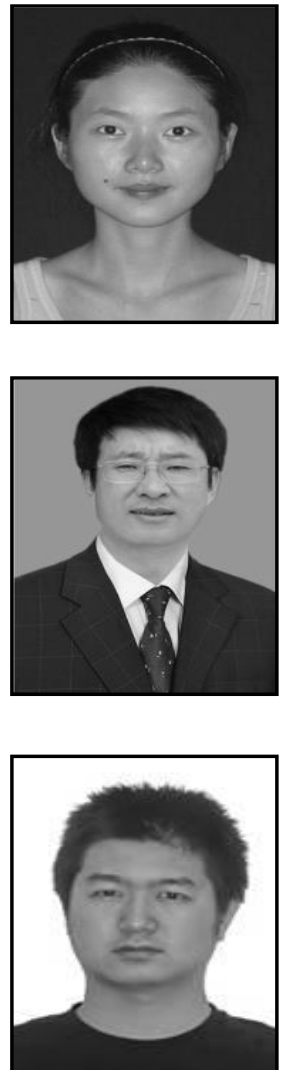

Lingli Zhao, She is a corresponding author, received the B.S. degree in Computer Science in 2001 from Central South University, and received M.S. and $\mathrm{PhD}$ degrees in Geographic Information System from Central South University, Changsha, China, in 2003, 2006 and 2011 respectively. Currently, she is working at School of Engineering of Honghe University, Mengzi, China. Her research interest covers 3D modeling, data integration and data mining.

Junsheng Li, received the B.S. degree in Physical in 1982 from Yunnan University, and received M.S. degree in information and electronic from Yunnan University, Kunming, China in 1997. Currently, he is working at School of Engineering of Honghe University, Mengzi, China. His research interest covers computer network.

Li Ma, He received the B.S. and PhD degrees in Photogrammetry and Remote Sensing from Wuhan University, Hubei, Chinain 2003 and 2011 respectively. From July 2004 to June 2011, he was a research associate at National Geomatics Center of China. Currently, he is working at Changjiang Institute of Survey, Planning, Design and Research, Wuhan, China. His research interests cover photogrammetry engineering and remote sensing applications. 
International Journal of Database and Theory and Application Vol.9, No.2 (2016) 